\title{
Um olhar implicado para os registros de mortes Indígenas no estado do Acre causadas por conta da Covid-19
}

\section{A look involved for the records of Indigenous deaths without Acre state caused by the Covid-19 container}

DOI: $10.46919 / \operatorname{archv2n3-007~}$

Recebimento dos originais: 01/05/2021

Aceitação para publicação: 30/06/2021

\author{
Valdemar Matos Paula \\ Graduando em Ciências Biológicas pelo Instituto Federal de Educação, Ciência e Tecnologia do Acre - \\ IFAC. \\ Endereço: Av. Brasil, 920, Xavier Maia. Rio Branco, AC. CEP: 69903-068. \\ E-mail: Vldmrmatos@gmail.com \\ Maria das Graças Alves Pereira \\ Psicóloga, Mestre em Medicina e Saúde, Doutora em Saúde Pública e professora EBTT do Instituto \\ Federal de Educação, Ciência e Tecnologia do Acre - IFAC. \\ Endereço: Av. Brasil, 920, Xavier Maia. Rio Branco, AC. CEP: 69903-068. \\ E-mail: Maria.pereira@ifac.edu.br
}

\section{RESUMO}

Os indígenas evidentemente necessitam da assistência de políticas públicas que busquem atender problemas relacionados a saúde pública dentro das aldeias e até mesmo fora delas, assim, discorrendo sobre os impactos causados por conta do agravamento da Covid-19 dentro destas comunidade, este estudo tem como finalidade apresentar os índices de mortes ocasionadas pela Covid-19 nas populações indígenas do Acre, ademais, o estudo fomenta a discussão sobre a relevância de novas ferramentas que otimizem a oferta de saúde pública para estes povos, especialmente, dentro da Amazônia. Optou-se por uma metodologia de busca pelos dados apresentados, logo, os dados foram coletados no banco de dados da Comissão Pró-Índio do Acre. Verificou-se que em dezembro do ano de 2020 foram registradas 27 mortes indígenas no Acre por conta da Covid-19 e os povos atingidos foram: Puyanawa - Jaminawas - Jaminawa Arara - Manxineru Huni kuî (Kaxinawa) - Madijá (Kulinas), Shawãdwa (Arara) - Shanenawa - Yawanawa - Nukini - Nawa Noke ko'í (Katukina) - Apolima Arara, ou seja, mais de 50\% dos povos indígenas do Acre. Pode ser concluindo que o estado do Acre necessita implantar políticas públicas que possam atender os povos indígenas de uma maneira eficaz e não esporádica, pois, o direito por saúde indígena deve existir. Ademais, é relevante que tais políticas públicas ocorram não apenas em tempos de pandemia, mas também, em período pós-pandêmico.

Palavras-chave: Covid-19, Estado do Acre, Indígenas, Amazônia Ocidental.

\begin{abstract}
The indigenous people evidently need the assistance of public policies that seek to address problems related to public health within the villages and even outside them, thus, talking about the impacts caused by the worsening of Covid-19 within these communities, this study aims to to present the death rates caused by Covid-19 in the indigenous populations of Acre, in addition, the study encourages discussion about the relevance of new tools that optimize the public health offer for these peoples, especially within the Amazon. We opted for a search methodology for the data presented, so the data were collected in the database of the Pró-Índio do Acre Commission. It was found that in December 2020, 27 indigenous deaths were recorded
\end{abstract}


in Acre due to Covid-19 and the affected peoples were: Puyanawa - Jaminawas - Jaminawa Arara Manxineru - Huni kuî (Kaxinawa) - Madijá (Kulinas), Shawãdwa (Macaw) - Shanenawa - Yawanawa Nukini - Nawa - Noke ko'í (Katukina) - Apolima Arara, that is, more than 50\% of the indigenous peoples of Acre. It can be concluded that the state of Acre needs to implement public policies that can serve indigenous peoples in an effective and non-sporadic way, since the right to indigenous health must exist. Furthermore, it is relevant that such public policies occur not only in times of pandemic, but also, in postpandemic period.

Keywords: Covid-19, State of Acre, Indigenous people, Western Amazon.

\section{INTRODUÇÃO}

O conhecimento a respeito da saúde, do histórico, e das relações sociais dos indígenas no Brasil é um conhecimento que existe desde a chegada dos europeus nas Américas, assim, através do que estes colonizadores puderam constatar em nossas terras, estima-se que no Brasil, eram residentes cerca de seis milhões de indígenas ainda no início do período histórico da colonização (BORGES et al., 2020).

No entanto, algumas etnias indígenas foram evidentemente exterminadas através de massacres, oriundos de lutas com o homem branco no ato de defender suas terras, como também, na recusa pelos diversos trabalhos forçados e epidemias que contribuíam para o agravamento do índice de mortes entre estes povos (CUNHA, 2012).

Tratando a respeito do estado do estado do Acre, um dos estados do norte do Brasil e localizado dentro da Amazônia, é possível afirmar que hoje existem aproximadamente 36 Terras Indígenas (TIs) que são homologadas pela FUNAI (Fundação Nacional do Índio) e que são devidamente reconhecidas pelo Governo Federal (ACRE, 2020; DO ACRE, 2020; PAULA, 2021).

Ressalta-se ainda que todas as TIs que existem dentro do território acreano, fazem fronteira ou localizam-se dentro de Unidades de Conservação, logo, esta observação fortalece o dever do estado em buscar o bem-estar destes povos, garantindo assim, direitos como educação e saúde.

Entretanto, é válido mencionar que os dados referentes a exata quantia dos povos indígenas no Acre, podem estar defasados e o real quantitativo pode ser ainda mais expressivos, visto que, nota-se que tanto o Governo Federal como o Governo estadual, não se empenham para que ocorra uma mensuração da real situação destes povos em todo o estado do Acre.

É sabido que devido a extensão territorial do estado, existe uma grande possibilidade que algumas TIs possam estar totalmente isoladas dentro do estado da Amazônia-acreana, neste sentindo, compreendese que deveria ser de total interesse do estado em ir em busca de ferramentas que pudessem atender as necessidades destes povos, incluindo assim, educação e saúde, especialmente, em tempos de Pandemia (DO ACRE, 2020). 
Os registros geográficos dos povos indígenas no estado do Acre estão distribuídos em 11 dos 22 munícipios, sendo possível perceber que estes povos ocupam uma área total de 2.436.811 ha, representando assim, cerca de 14,6\% do território acreano (DO ACRE, 2020; PAULA, 2021; BORGES et al., 2020). Ademais, no Acre habitam 15 povos indígenas, originários das três famílias linguísticas (Pano, Aruak e Arawa).

Neste sentido, com o agravamento da Pandemia do novo Coronavírus no mundo e após a constatação de que os sintomas causados por este vírus podem ser determinantes para a mudança do quadro de saúde dos seres humanos podendo causar até mesmo mortes, diversos países apresentaram uma mobilização para que ocorram medidas que possam atuar na disseminação deste vírus (WEI et al., 2020).

Contudo, em nosso país, o Brasil, durante diversos momentos da pandemia foi possível perceber que nosso principal líder político não tratou a crise sanitária como deveria, o que contribuiu para o triste registro de mais de 200 mil mortes incluindo indígenas, por conta deste vírus (BITTENCOURT, 2020).

Além de evidente índice de morte em nossa sociedade causar preocupação nos órgãos sanitários do país e do mundo, é relevante a existência de um olhar mais rigoroso para os povos indígenas, especialmente os que vivem na Amazônia em localidades de difícil acesso, visto que, estes povos não possuem assistência básica de saúde significante, o que permite a inferência de que o registro de patologias como a COVID-19 em TIs pode ser determinante para que ocorra uma extinção em massa de inúmeros indígenas (BORGES et al., 2019; PIMENTA, 2009; COIMBRA et al., 2003).

Neste sentindo, a existência de um monitoramento, por parte do estado, mais rígido da ocorrência e registro dos agravamentos causados por conta da COVID-19 nas TIs no Acre, deve ocorrer de forma mais eficaz, visto que, a alta concentração de povos indígenas em suas comunidades locais e na maioria das vezes, isoladas, pode ser propícia para um vírus que apresenta uma taxa alta de transmissibilidade, algo que contribui para que o cenário seja devastador para estes povos (BERNARDI \& LA TORRE, 2020).

Segundo dados divulgados por Do Acre (2020) o estado do Acre já apresentava no segundo semestre de 2020, uma taxa de mortandade bem preocupante em TIs referente aos casos de COVID-19, e, a quantidade de mortes de povos indígenas em todo território acreano causa preocupação em diferentes aspectos humanísticos, sanitários e étnicos, visto que, em tempos de Pandemia é essencial que o estado busque ações que possam mitigar os danos causados dentro destas comunidades tradicionais (PAULA, 2021).

Baseando-se nisto, este estudo tem como objetivo apresentar os registros de mortes ocasionadas pela COVID-19 em todo território do Acre dentro das TIs, mortes registradas ao longo da Pandemia, mas que foram divulgadas pela comissão pró-índio do estado do Acre no mês de dezembro do ano de 2020, ademais, este estudo busca discutir sobre a necessidade de existirem políticas públicas que priorizem a 
promoção de saúde pública para os povos indígenas do Acre e da Amazônia, principalmente em tempos de pandemia.

\section{MATERIAL E MÉTODOS}

Para a realização desta pesquisa adotou-se uma metodologia de busca pelos dados no banco de dados da Comissão Pró-Índio do Acre - (https://cpiacre.org.br/). Assim, foram coletados os dados referentes as mortes indígenas no estado do Acre durante a pandemia da COVID-19, especialmente dados divulgados no mês de dezembro de 2020, logo, os dados apresentados neste estudo, possivelmente, já se encontram bem mais expressivos, pois, a COVID-19 continua causando mortes dentro da nossa sociedade.

A busca pelos dados ocorreu de acordo com as seguintes etapas: Houve um acesso na página da Comissão Pró-Índio do Acre e em seguida foi selecionada o item com as informações mais detalhadas sobres as TIs, extensão, população, município, povo, número de aldeias, número de Agentes Indígenas de Saúde - AIS e de Agentes Indígenas de Saneamento - AISAN e foi verificado os casos acumulados de COVID-19 nas TIs onde o coronavírus chegou e a quantidade de óbitos ocasionados por conta da COVID19.

Pelo fato de que a coleta de dados ocorreu em um banco de dados de domínio público, este estudo não tem a necessidade de ser submetido ao Comitê de Ética e Pesquisa, pois, segundo a Resolução $\mathrm{n}^{\circ} 510$ de 7 de abril de 2016, a retirada de informações de domínio público e pesquisa com banco de dados, sem a identificação individual, não tem a necessidade da avaliação do sistema CEP/CONEP (BRASIL, 2016b). Desta forma, os dados foram coletados durante o mês de dezembro de 2020 e analisados para que existisse uma discussão relevante sobre o tema na exposição deste estudo.

\section{O REFLEXO DA COVID-19 DENTRO DO TERRITÓRIO DOS POVOS INDÍGENAS E AS IMPLICAÇÕES PARA ESTES POVOS}

A Amazônia é caracterizada por sua diversidade, desde a fauna e a flora até a exuberância de seus rios, no entanto, tais características contribuem para que ocorram uma logística complexa para que a promoção de educação, saúde e outros direitos dos povos da floresta sejam entregues de forma eficaz (PAULA et al., 2020; PAULA, 2021; GIATTI \& CUTOLO, 2012).

Desta forma, é essencial que o Estado atue de forma persistente dentro destas localidades e que sejam considerados todos os percalços evidentes na busca pela promoção de saúde pública.

Segundo Santos et al., (2020) tratando em seu estudo respeito de patologias em indígenas que residem especialmente na Amazônia, este autor relata que historicamente, algumas doenças respiratórias com aspectos de infecções contagiosas, como uma simples gripe, podem ser consideradas como grandes ameaças para os indígenas, e, isso ocorre por conta de que os indígenas apresentam uma baixa imunidade. 
De acordo com Bernardi \& La Torre (2020) a Organização dos Estados Americanos emitiu um alerta referente ao cenário encontrado entre os povos indígenas, ou seja, estes povos convivem com uma dupla vulnerabilidade evidenciada por conta do isolamento geográfico de grande parte das TIs da Amazônia, mas também, de uma latente marginalização histórica (OLIVEIRA et al., 2020).

A despeito dos indígenas que vivem na Amazônia, sejam índios isolados ou que já possuam um convívio com as grandes cidades urbanas, estes povos ainda enfrentam reincidentes ameaças que violam seus direitos, como por exemplo, o índice significante de desmatamento, a degradação dos ecossistemas que estes povos estão inseridos e uma extração de recursos naturais sem uma eficiente fiscalização, contribuindo assim, para uma desfragmentação das TIs (PEREIRA et al., 2021).

Porém, neste momento cheio de percalços para a saúde pública e para a toda a sociedade do nosso país, os povos indígenas ainda necessitam lutar contra os efeitos da pandemia da COVID-19 como também, medidas associadas ao ato de paralisar o avanço de contaminação e mortes causadas por este vírus, entre os indígenas.

Nesta ótica, as autoridades do nosso país necessitam reavaliar a prioridade existente para o bemestar e a promoção eficiente de políticas públicas que possam garantir a saúde das populações indígenas da Amazônia. Ademais, nossas autoridades sanitárias devem ter em mente um melhor plano de combate contra o coronavírus e uma atuação firme na oferta de saúde para os povos indígenas, já imaginando um período de pós-pandemia, é relevante a compreensão e aceitação de que a grande maioria dos estados do norte do Brasil, possuem seus sistemas de saúde frágeis e o Estado não assume suas obrigações referentes a proteção das TIs (FELLOWS et al., 2020).

Logo, para que exista uma atuação dentro das TIs, é primordial que ocorram políticas públicas que priorizem a saúde e os hábitos sociais dos povos indígenas da Amazônia, desta forma, é relevante afirmar que, por conta do cenário vivido entre os povos indígenas da Amazônia, muitos povos idealizaram medidas de isolamento, por conta própria, visando uma proteção contra a COVID-19, assim, TIs que possuíam hábitos turísticos, visitas de pessoas do exterior ou de outras comunidades, deixaram de receber visitas para proteger a todos (BRAGATO et al., 2021).

Contudo, ainda existindo algumas medidas dos próprios indígenas e em alguns momentos do Estado, nota-se que na região Amazônica, especialmente em comunidades ribeirinhas e TIs a COVID-19 apresenta-se de forma letal e contagiosa e os registros de mortes nas TIs de todos os estados do Norte comprovam isto, logo, estes dados corroboram o quanto os povos indígenas necessitam de uma atenção mais significativa (FELLOWS et al., 2020; RIBEIRO, 2020). 


\section{A SITUAÇÃO DO ACRE E UMA REFLEXÃO NA AMAZÔNIA}

É válido mencionar, que os dados apresentados neste estudo são dados que representam como o estado do Acre finalizou o ano de 2020 no cenário de mortalidade indígenas por conta da COVID-19, assim, é possível afirmar que se compararmos os dados deste estudo com dados atuais, os valores serão mais expressivos e irão comprovar que os casos de COVID-19 e mortalidade entre os povos indígenas serão maiores, corroborando a preocupação apresentada no estudo e a fala de que é necessário um olhar implicado para esta situação.

De acordo com a última atualização dos dados da Comissão Pró-índio do Acre, o Acre registrou no mês de dezembro de 2021, 2375 casos de COVID-19 entre os indígenas, no entanto, esses casos acumulados referem-se a indígenas residentes de TIs e residentes nos munícipios, assim, são 1209 casos de indígenas com COVID-19 nas TIs e 1166 com COVID-19 nos munícipios do estado do Acre, como apresenta o mapa 1.

É válido mencionar que possivelmente estes dados possam estar um status de subnotificação, visto que, nem sempre ocorre um emprenho efetivo dos órgãos de saúde para que real situação possa ser analisada.

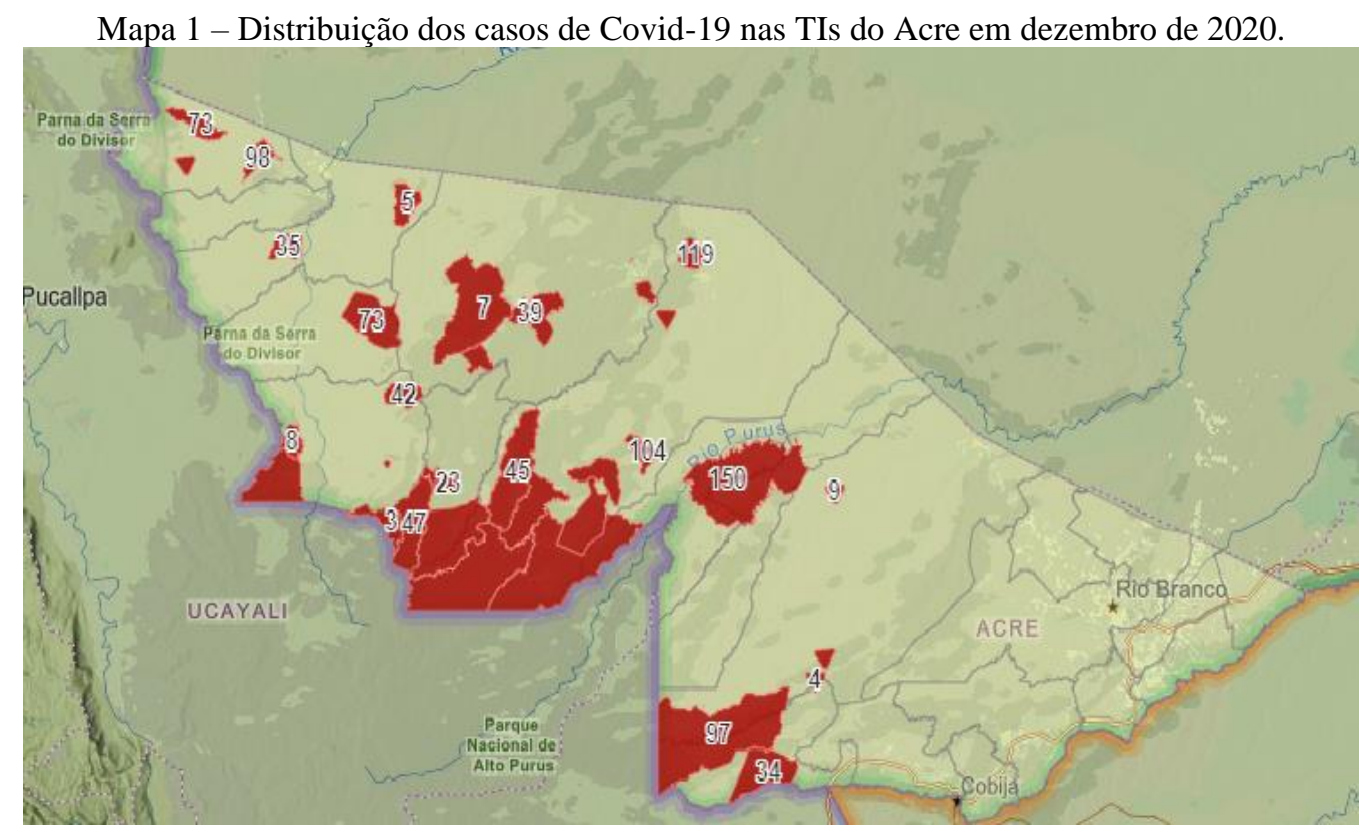

Fonte: SESACRE-Covid19.ac.gov.br/monitoramento, SESAI-DSEI ARJ/ARPU, COIAB, 2020.

O estado do Acre possui uma Casa da Saúde Indígena (CASAI) que se constitui parte do Subsistemas de Atenção à Saúde Indígena (SASI), e este local, é responsável pelo suporte aos indígenas durante a busca por tratamentos de doenças, no entanto, este local de atendimento localiza-se na capital do estado. Ademais, a CASAI do Acre, auxilia indígenas dos Distritos Sanitários Especiais Indígenas (Dsei) do Alto Rio Purus e do Alto Rio Juruá (DANTAS, 2010). 
Contudo, no mapa 1 é possível perceber que a distribuição dos casos de COVID-19 no estado do Acre, apresentam-se de uma maneira evidente nas aldeias de difícil acesso e em áreas afastas da capital do estado, algo que contribui para que em casos mais graves, indígenas possam chegar ao óbito antes de conseguirem o atendimento médico nos munícipios ou na capital do estado.

Segundo Bernardi \& La Torre (2020) grande parte dos países latino-americanos não consideram e não apresentam ações efetivas para o combate da COVID-19 nas TIs, e, esta situação é percebível quando analisamos os índices referentes a mortalidade indígena encontrados no estado do Acre, pois, estes corroboram com a fala destas autoras e evidenciam que é essencial um plano mais efetivo para estes povos.

Um aspecto relevante para mencionar quando analisamos medidas de isolamento destes povos, é o fato de que os povos indígenas Yawanawa, tiveram iniciativas de isolamento e restrições ao acesso da TI (DO ACRE, 2020), porém, tais medidas não foram suficientes para que indígenas desta etnia e de outras fossem contaminados e até mesmo mortos por conta da COVID-19, pois os casos e mortes ocorreram dentro da TI.

Desta forma, afirma-se que políticas de cunho ambiental e ações que possuem a finalidade de assegurar os direitos como a saúde dos povos da floresta, foram deixadas de lado e o termo "Passar a boiada" foi cunhado e mentalizado por nossos governantes, deixando claro que a oferta de saúde para estes povos e a preservação da floresta e consequentemente as TIs não é prioritária neste momento.

A COICA (Coordenadoria das Organizações Indígenas da Bacia da Amazônia) divulgou um documento público pedindo que houvesse medidas para a proteção dos povos indígenas e consequentemente para que existissem ações de prevenções que evitasse a infecção em massa e ações que garantissem o acesso destes povos aos serviços de saúde dentro dos territórios indígenas (GÁMEZ, 2020).

Desta forma, se estas medidas estivessem sendo adotadas desde o início da pandemia, possivelmente, algumas mortes teriam sido evitadas e a taxa de mortalidade diminuída, não só do estado do Acre, mas também de outras TIs da Amazônia, visto que, as doenças respiratórias são as principais razões das mortes evidente nas populações nativas do Brasil, logo, a existência de políticas públicas que garantissem os direitos sanitários destes povos, deveriam existir com uma maior frequência e eficácia.

Tratando a respeito do número de óbitos dentro das TIs, os dados chegam a 27 mortes e já somam 13 povos atingidos pelo novo coronavírus, como podemos observar no mapa 2. 
Mapa 2 - Distribuição dos óbitos por Covid-19 nas TIs do Acre em Dezembro de 2020.

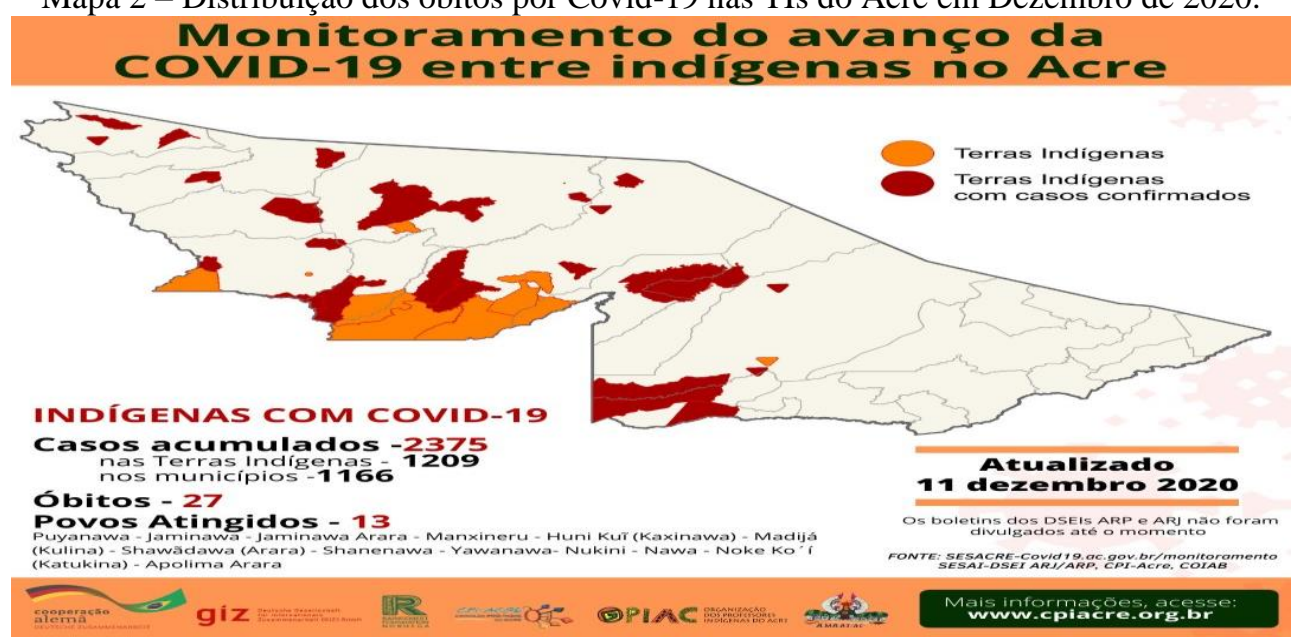

Fonte: SESACRE-Covid19.ac.gov.br/monitoramento, SESAI-DSEI ARJ/ARPU, COIAB, 2020.

Um dado que chama atenção ao analisar o mapa 2 é o valor bem significante expressado pelos casos de mortes, 27, em todo o estado do Acre, no entanto, é essencial a existência de estudos futuros para que ocorra um levantamento de possíveis doenças preexistentes nesses indígenas ou a não existência de doenças preexistentes, dessa forma, o entendimento do agravamento da saúde destes povos poderá ocorrer com mais familiaridade, e, a disseminação das mortes por COVID-19, podem ser analisadas na tabela 1.

Tabela 1. TIs que tiveram registros de mortes no Acre.

1. Puyanawa - 2. Jaminawas - 3. Jaminawa Arara - 4. Manxineru - 5. Huni kuî (Kaxinawa) - 6. Madijá (Kulinas) 7.Shawãdwa (Arara) - 8. Shanenawa - 9. Yawanawa - 10. Nukini - 11. Nawa - 12. Noke ko'í (Katukina) - 13. Apolima Arara.

Fonte: SESACRE-Covid19.ac.gov.br/monitoramento, SESAI-DSEI ARJ/ARPU, COIAB, 2020.

Nota-se que mais de $50 \%$ dos povos indígenas do Acre foram atingidos, assim, a existência de políticas públicas que ofereçam saúde de qualidade para estes povos é evidentemente necessária não somente em tempos de pandemia, como também, pós-pandemia, considerando que estes povos possuem o direito de seres assistidos em suas necessidades, em especial, na saúde destes povos.

O momento em que vivemos servirá como reflexão para que novas ações possam ocorrer em busca de melhores condições para estes povos, desta forma, o estado deve se manifestar para mitigar os danos causados pela COVID-19 dentro destas comunidades indígenas.

Ademais, é relevante que exista uma atenção para o atual modelo de atenção para a saúde destes povos, pois, o agravamento dos casos de COVID-19 dentro destas comunidades pode significar um desastre para os povos tradicionais que já lutam para sobreviver em meio à uma sociedade que não valoriza os povos indígenas. 
$\mathrm{O}$ índice alto de mortes dentro das aldeias indígenas e até mesmo fora das aldeias, nos permite afirmar que existe uma vulnerabilidade destes povos dentro do estado do Acre e que a assistência referente aos cuidados com a saúde destes povos, é ineficaz.

Outrossim, a taxa de mortes entre os indígenas possui uma relação bem evidente com a existência ou inexistências de ações públicas que busquem sanar as dificuldades de povos que residem na capital, nos municípios e até mesmo isolados dentro da floresta.

\section{CONSIDERAÇÕES FINAIS}

Os registros de mortes por conta da COVID-19 entre os indígenas no Acre e na Amazônia, representa não somente índices de falta de ações para a saúde dos povos indígenas, mas também, evidentes percalços na divulgação para toda a sociedade, visto que, é evidente que os dados podem ser divulgados com maiores esforções para a melhoria da qualidade dos registros e do conhecimento de que esses povos necessitam ser assistidos.

Ademais, o estado do Acre necessita implantar políticas públicas que possam atender os povos indígenas de uma maneira eficaz e não esporádica, pois, o direito por saúde indígena é garantido por lei para estes povos, e, tais medidas não devem ocorrer somente em tempos de Pandemia, mas de forma contínua.

É necessário ainda, o incentivo de estudos como estes que busquem apresentar de forma significante o quanto estes povos carecem de uma atenção referente à saúde de qualidade dentro e fora das aldeias.

Assim, o perfil das populações indígenas na Amazônia Ocidental só será visto com um outro olhar pelo mundo, quando o estado se preocupar em promover ações eficazes para estes povos. 


\section{REFERÊNCIAS}

ACRE. GDEDA. Terras Indígenas. Disponível em: http://www.ac.gov.br/br/. Acesso em: 31 de dez. 2020.

BERNARDI, P.; LA TORRE, D. de. Covid-19: Uma ameaça a mais para a Amazônia. 2020.

BITTENCOURT, R. N. Pandemia, isolamento social e colapso global. Revista Espaço Acadêmico, v. 19, n. 221, p. 168-178, 2020.

BORGES, M. F. de. S. O.; SILVA, I. F. da.; KOIFMAN, R. Histórico social, demográfico e de saúde dos povos indígenas do estado do Acre, Brasil. Ciência \& Saúde Coletiva, v. 25, p. 2237-2246, 2020.

BORGES, M. F. de. S. O. et al. Cancer mortality among indigenous population in Acre State, Brazil. Cadernos de saude publica, v. 35, n. 5, 2019.

BRASIL. Ministério da Saúde. Conselho Nacional de Saúde. Resolução no 510, de 07 de abril de 2016 b. Dispõe sobre as normas aplicáveis a pesquisas em Ciências Humanas e Sociais. Brasília: Conselho Nacional de Saúde, 2016b. Disponível em: http://conselho.saude.gov.br/resolucoes/2016/2016/Reso510.pdf. Acesso em: 28 de abr. 2021.

BRAGATO, F. F.; RIOS, R. R.; BERNARDI, B. B. COVID-19 e os indígenas no brasil: proteção antidiscriminatória étnico-racial e direitos de minorias. Veredas do Direito: Direito Ambiental e Desenvolvimento Sustentável, v. 18, n. 40, 2021.

COIMBRA, J. R.; CARLOS, E. A.; SANTOS, R. V.; ESCOBAR, A. L. Epidemiologia e saúde dos povos indígenas no Brasil. Editora Fiocruz, 2003.

CUNHA, M. C. da. Índios no Brasil: História. Direitos e Cidadania. São Paulo: Claro Enigma, 2012.

DANTAS, F. L. L. Perfil de morbidade da população indígena infantil referenciada para a Casa de Saúde Indígena (CASAI) de Rio Branco. 2010. Tese de Doutorado. Universidade de São Paulo.

DO ACRE, C.P.Í., et al. Cresce casos de indígenas com coronavírus no Acre. 2020.

DO ACRE, C.P.Í., et al. Indigenista Meirelles fala sobre risco de Covid-19 entre povos isolados. 2020.

DO ACRE, C. P. Í. et al. Contra o Coronavírus, Fica na Aldeia!. 2020.

FELLOWS, M. et al. Não são número, são vidas!: A ameaça da Covid-19 aos povos indígenas na Amazônia Brasileira. 2020.

GÁMEZ, L. A dupla ameaça para os povos da Amazônia. 2020.

GIATTI, L. L.; CUTOLO, S. A. Acesso à água para consumo humano e aspectos de saúde pública na Amazônia Legal. Ambiente \& Sociedade, v. 15, n. 1, p. 93-109, 2012.

OLIVEIRA, U. et al. Modelagem da vulnerabilidade dos povos indígenas no Brasil ao covid-19. Instituto Socioambiental, 2020. 
PEREIRA, L. I.; DE FREITAS COCA, E. L.; ORIGUÉLA, C. F. O" PASSAR A BOIADA" NA QUESTÃO AGRÁRIA BRASILEIRA EM TEMPOS DE PANDEMIA/The "passar a boiada" on the Brazilian agrarian question in times of pandemic/El" passar a boiada" sobre la cuestión agraria brasileña en tiempos de pandemia. REVISTA NERA, n. 56, p. 8-23, 2021.

PAULA, V. M. Reflexões sobre os povos indígenas e a necessidade de existirem promoções de políticas públicas em tempos de pandemia: relato da situação no Acre, Brasil. Revista em Favor de Igualdade Racial, v. 4, n. 1, p. 179-190, 2021.

PAUlA, V. M.; PEREIRA, M. das. G. A.; SOARES, J. R. A promoção de educação do campo na Amazônia-acreana através do Asas da Florestania. Educamazônia-Educação, Sociedade e Meio Ambiente, v. 24, n. 1, jan-jun, p. 58-69, 2020.

PIMENTA, J. Povos indígenas, fronteiras amazônicas e soberania nacional. Algumas reflexões a partir dos Ashaninka do Acre. In: Proceedings from the 61st Annual Meeting of the Brazilian Society for Scientific Progress: Amazon Science and Culture. Round Table: Indigenous Groups in Amazonia. Manaus, Brazil. (http://www. sbpcnet. org. br/livro/61ra/mesas_redondas/MR_JosePimenta. pdf). Accessed. 2009.

RIBEIRO, M. F. Coronavírus já atinge 18 etnias no país, nas aldeias e nas cidades. 2020.

SANTOS, R. V.; PONTES, A. L.; COIMBRA, J. R.; CARLOS, E. A. Um "fato social total": COVID-19 e povos indígenas no Brasil. 2020.

WEI, H. et al. The 2019 novel cornoavirus pneumonia with onset of oculomotor nerve palsy: a case study. Journal of neurology, v. 267, n. 5, p. 1550-1553, 2020. 